\title{
Electric and magnetic spectra from MHD to electron scales in the magnetosheath
}

\author{
L. Matteini, ${ }^{1 \star}$ O. Alexandrova, ${ }^{2}$ C. H. K. Chen ${ }^{1}$ and C. Lacombe ${ }^{2}$ \\ ${ }^{1}$ Department of Physics, Imperial College London, London SW7 2AZ, UK \\ ${ }^{2}$ LESIA-Observatoire de Paris, PSL Research University, CNRS, UPMC Université Paris 06, Université Paris-Diderot, \\ 5 place Jules Janssen, F-92190 Meudon, France
}

Accepted 2016 December 2. Received 2016 December 2; in original form 2016 July 27

\begin{abstract}
We investigate the transition of the turbulence from large to kinetic scales using Cluster observations. Simultaneous spectra of magnetic and electric fields in the Earth's magnetosheath from magnetohydrodynamic (MHD) to electron scales are presented for the first time. While the two spectra have approximatively similar behaviour in the fluid-MHD regime, they show different trends in the kinetic range. As the magnetic field spectrum steepens at ion scales, the electric field spectrum is characterized by a shallower power law continuing down to electron scales. Such an evolution is consistent with theoretical expectations, assuming that the turbulence is dominated by highly oblique $\boldsymbol{k}$-vectors and that between ion and electron scales the electric field is governed by the non-ideal terms in the generalized Ohm's law. This leads to an expected linear increase of the electric-to-magnetic ratio of fluctuations, consistent with observations presented here. The influence of local whistler wave activity on electron-scale spectra is also discussed.
\end{abstract}

Key words: magnetic fields - MHD - plasmas - turbulence - solar wind.

\section{INTRODUCTION}

Turbulence is a ubiquitous phenomenon in space and astrophysical plasmas. Through an energy cascade connecting the many scales of the system (fluid, ion, and electron), turbulence plays an important role in setting the properties of energy transport and dissipation. In collisionless plasmas, turbulence is believed to be the process bringing energy from the large characteristic fluid scales of the system down to small kinetic scales where it can be efficiently dissipated by the direct interaction of particles with electromagnetic fields, causing the heating of the plasma. The nature of these processes is still debated and is relevant for the modelling of many astrophysical systems, like the solar corona (Rappazzo, Velli \& Einaudi 2013), Earth (Alexandrova et al. 2006; Sahraoui et al. 2006) and planetary (Alexandrova \& Saur 2008; von Papen, Saur \& Alexandrova 2014; Hadid et al. 2015) magnetospheres, sheared accretion discs (Kunz, Schekochihin \& Stone 2014), and the hot intracluster medium (Schekochihin et al. 2005). The solar wind constitutes an exceptional laboratory for plasma turbulence (Alexandrova et al. 2013; Bruno \& Carbone 2013). At large scales, where the motion of the plasma is essentially that of a single fluid (no macroscopic drifts between ions and electrons), the dominant Alfvénic fluctuations observed over a wide range of scales can be described in the framework of incompressible magnetohydrodynamics (MHD) and display a typical power-law spectrum with index between $-5 / 3$ and $-3 / 2$ characteristic of a turbulent regime (e.g. Chen et al. 2013b).

As the cascade approaches kinetic scales, some changes occur. Note that in order to highlight the different ranges of scales involved in the cascade, we use here the term kinetic to describe aspects of the turbulence occurring at wavenumbers $k$ associated to particle typical scales (the ion and electron inertial length and Larmor radius, $d_{\mathrm{i}, \mathrm{e}}$ and $\rho_{\mathrm{i}, \mathrm{e}}$, respectively). However, in the following we will consider physical properties that are still related to the collective multifluid behaviour of the plasma, and not to intrinsically kinetic processes (e.g. wave-particle interactions and associated damping). In situ observations clearly show a steepening of the magnetic field spectrum (e.g. Leamon et al. 1998), as well as of velocity and density spectra (Chen et al. 2012; Šafránková et al. 2013), signatures of the transition from a fluid-MHD regime to a kinetic one.

However, the evolution of the electric field spectrum associated with the fluctuations as the cascade reaches ion scales is not well determined yet from observations. Previous works (Bale et al. 2005; Sahraoui et al. 2009; Salem et al. 2012) suggest a flattening of the electric field spectrum around the proton gyroradius/inertial length, together with a monotonic increase of the electric-to-magnetic ratio $E / B$. However, the change in the slope occurs very close to the electric field instrument noise threshold for typical solar-wind conditions, so that it is not possible to draw a firm conclusion about the observed behaviour. The next generation of spacecraft with higher resolution instruments on board will make the comparison possible. 
There is however a region accessible to in situ explorations where a detailed analysis of electric and magnetic spectra at kinetic scales can be performed, using current data; this is Earth's magnetosheath. The power of the turbulence downstream of the Earth's bow shock is higher than in the solar wind, allowing us to access a wider range of frequencies. Spectra of the magnetic field from MHD to sub-ion scales in the magnetosheath have been reported by Alexandrova, Lacombe \& Mangeney (2008a) using the Cluster spacecraft, and on kinetic scales by e.g. Sundkvist et al. (2007) and Huang et al. (2014); see also Zimbardo et al. (2010) for a review on magnetic turbulence in this region. Properties of electric and magnetic spectra between ion and electron scales have been previously studied by Mangeney et al. (2006) and Lacombe et al. (2006), confirming that this regime can be well resolved in the magnetosheath using Cluster. However, these latter studies have focused only on data from the high-frequency part of the spectrum. In this work, we study the full transition from MHD to kinetic regime of plasma turbulence in the magnetosheath, extending previous works and comparing for the first time electric and magnetic spectra all the way down from fluid to electron scales.

\section{THEORETICAL PREDICTION}

In the sub-ion range $\left(k d_{\mathrm{i}}>1\right)$, a shallower electric field spectrum, with respect to magnetic is expected, leading to an increase in the ratio of electric-to-magnetic fluctuations $\delta E / \delta B \propto k d_{\mathrm{i}}$. This is a direct consequence of the nature of the turbulent fluctuations at such small scales (see e.g. Schekochihin et al. 2009; Boldyrev et al. 2013, for a comprehensive and detailed description of the properties of sub-ion scale turbulence); we give here a simple derivation of such a scaling, based on the generalized Ohm's law and with inputs from in situ observations. In the inertial range of solar-wind turbulence far from the ion scales - the relation between electric and magnetic field is controlled by the ideal-MHD term: $\boldsymbol{E}=-\boldsymbol{V} \times \boldsymbol{B}$. At these scales the cascade is dominated by transverse Alfvénic fluctuations and, as a consequence, in the plasma frame (at rest with the flow), fluctuations in the electric field are related to the transverse velocity field $\delta E \sim \delta V_{\perp} B_{0}$. It can be shown that the electric spectrum then essentially follows the velocity spectrum (Chen et al. 2011). Such a picture changes when approaching kinetic scales with the breakdown of the ideal-MHD assumption. At scales where the differential motion of ions and electrons becomes important, one has to consider the generalized Ohm's law in describing the electric field. Assuming isotropic electrons and neglecting their inertia, this reads

$\boldsymbol{E}=-\boldsymbol{V} \times \boldsymbol{B}+\frac{\boldsymbol{J} \times \boldsymbol{B}}{e n}-\frac{\nabla P_{\mathrm{e}}}{e n}$,

where $\boldsymbol{V}$ now refers to proton velocity, highlighting effects due to ion-electron decoupling leading to the Hall term $\boldsymbol{J} \times \boldsymbol{B} \propto$ $(\nabla \times \boldsymbol{B}) \times \boldsymbol{B} \propto B_{0} \nabla \delta B$. We assume here that transverse and fieldaligned magnetic fluctuations have approximatively the same amplitude $\delta B_{\perp} \sim \delta B_{\|} \sim \delta B$ as this is supported by in situ observations of both solar wind (Alexandrova et al. 2008b; Kiyani et al. 2013) and magnetosheath (Alexandrova et al. 2008a) at scales where the Hall effect becomes important. The last term of equation (1) is related to the electron pressure $P_{\mathrm{e}}=n k_{\mathrm{B}} T_{\mathrm{e}}$, where $n$ and $T_{\mathrm{e}}$ are electron density and temperature. If we assume isothermal electrons (note that this assumption is usually well justified in space plasmas; see e.g. Schekochihin et al. 2009) or a simple functional form as a polytropic law, this term is directly related to density fluctuations $\sim \nabla n$, which are considered equal for electron and ions to ensure quasi-neutrality. It is known that in the solar wind, density fluctuations become comparable to magnetic ones at small scales (Chen et al. 2013a) and thus the last term of equation (1) cannot be neglected at sub-ion scales.

In order to apply such a description to spacecraft observations, some assumptions are in order here: first is that measured spectra correspond to spatial variations in the plasma (Taylor hypothesis), as the speed of the flow is much larger than the typical phase velocity of the fluctuations. This means that frequency spectra collected by a spacecraft can be considered wavenumber spectra, according to a suitable Doppler-shift $k=2 \pi f / V_{\text {Sw. Secondly, as proposed }}$ in theoretical works (Cho \& Lazarian 2004) and in agreement with in situ measurements (Lacombe et al. 2006; Mangeney et al. 2006; Chen et al. 2010), the turbulence characterizing the plasma at these scales is mainly constituted by highly oblique $\boldsymbol{k}$-vectors $\boldsymbol{k} \sim \boldsymbol{k}_{\perp}$. This means that the relevant gradients in the operators of equation (1) are the perpendicular ones $\left(\nabla_{\perp}\right)$. In this framework, it is possible to show that when non-ideal terms in the Ohm's law dominate in equation (1), so that the first term on the right-hand side can be neglected (i.e. when proton-velocity fluctuations $\delta V$ decouple from the magnetic field and $\delta V<\delta B$, as expected at sub-ion scales) and if restricting the analysis to quasi-perpendicular transverse fluctuations, the expected electric field scales as (e.g. Franci et al. 2015b)

$e n \delta E \sim B_{0} \nabla_{\perp} \delta B+T_{\mathrm{e}} \nabla_{\perp} \delta n \propto \nabla_{\perp}\left(\frac{\delta B}{B_{0}}+\beta_{\mathrm{e}} \frac{\delta n}{n}\right)$.

Finally, in situ measurements in the solar wind demonstrate that magnetic and density fluctuations follow approximatively the same power law $\delta B(k) \sim \delta n(k)$ between ion and electron scales (Chen et al. 2012). This observational result is in very good agreement with theoretical expectations for low-frequency turbulence in the sub-ion regime (Schekochihin et al. 2009) and kinetic simulations where all the terms of equation (1) are taken into account (Franci et al. 2015b). Within this additional assumption, it is then possible to simply consider at sub-ion scales a relation $\delta E \propto \nabla_{\perp} \delta B$ between $\delta E$ and $\delta B$ from equation (2) leading to the asymptotic trend in $\boldsymbol{k}$-vector space:

$\frac{\delta E^{\text {kin }}}{V_{\mathrm{A}}} \sim k d_{\mathrm{i}} \delta B$,

where we have introduced the Alfvén speed $V_{\mathrm{A}}$ and the proton inertial length $d_{\mathrm{i}}$ as characteristic velocity and spatial scales, respectively. Note that, alternatively, one could introduce the ion Larmor radius, $\rho_{\mathrm{i}}=d_{\mathrm{i}} \sqrt{\beta_{\mathrm{i}}}$, where $\beta_{\mathrm{i}}$ is the ion plasma beta. In equation (3), we label as kinetic the contribution to the electric field supported by the non-ideal terms in equation (1), as this is expected to be the dominant contribution at small scales. This is the relation that we want to test in the sub-ion regime in this work.

For the reader interested in the quantitative estimation of the coefficients linking different fluctuating quantities, these can be found in Schekochihin et al. (2009) and Boldyrev et al. (2013), and depend on the local plasma beta and the electron-to-proton temperature ratio. In this framework, predictions from kinetic Alfvén wave (KAW) turbulence seem to be in good agreement with both solar-wind observations (Chen et al. 2013b) and non-linear numerical simulations of sub-ion turbulence (e.g. Howes et al. 2011; Franci et al. 2015a). Note however that recent observational (Chen et al. 2014) and numerical (Cerri et al. 2016; Franci et al. 2016) investigations suggest that the quantitative agreement with KAW predictions is fulfilled better at high ( $\gtrsim 1)$ than low beta; this aspect will be the subject of a forthcoming study.

Electric field measurements presented in this analysis correspond to spacecraft frame observations $\hat{\boldsymbol{E}}=\boldsymbol{E}-\boldsymbol{V}_{0} \times \boldsymbol{B}$. Then, $\hat{\boldsymbol{E}}$ 
fluctuations are expected to be larger than those of the real plasma frame electric field $\boldsymbol{E}$ due to the motional contribution $\sim V_{0} \delta B$, where $V_{0}$ is the flow speed. Separating the ideal $\left(E^{\mathrm{MHD}}\right)$ and nonideal $\left(E^{\mathrm{kin}}\right)$ contributions, one has in the spacecraft frame

$\delta \hat{\boldsymbol{E}}=\delta \boldsymbol{E}^{\mathrm{MHD}}+\delta \boldsymbol{E}^{\mathrm{kin}}-\boldsymbol{V}_{0} \times \boldsymbol{B}$.

Expanding the different terms recalling that at MHD scales $\delta E^{\mathrm{MHD}} \sim \delta V B_{0}$ and using equation (3), we obtain

$\delta \hat{E} \sim \delta V B_{0}+k d_{\mathrm{i}} V_{\mathrm{A}} \delta B-V_{0} \delta B$.

Dividing by the Alfvén speed and assuming that at large scale for Alfvénic fluctuations $\delta V / V_{\mathrm{A}} \sim \delta B / B_{0}$, this finally reads

$\frac{\delta \hat{E}}{V_{\mathrm{A}}} \sim \delta B+k d_{\mathrm{i}} \delta B-\delta B \frac{V_{0}}{V_{\mathrm{A}}}=\delta B\left(1+k d_{\mathrm{i}}-\frac{V_{0}}{V_{\mathrm{A}}}\right)$.

At large scales $\left(k d_{\mathrm{i}} \ll 1\right)$, when the ideal term dominates, such a contribution is

$$
\frac{\delta \hat{E}^{\mathrm{MHD}}}{V_{\mathrm{A}}} \sim \delta B\left(1-\frac{V_{0}}{V_{\mathrm{A}}}\right),
$$

thus increased by a quantity $\sim \delta B V_{0} / V_{\mathrm{A}}$ with respect to the plasma frame $\left(V_{0}=0\right)$. As typically $V_{0} \sim 10 V_{\mathrm{A}}$ at 1 au, then $\delta \hat{E}^{\mathrm{MHD}} \gg$ $V_{\mathrm{A}} \delta B$ in the solar wind (Chen et al. 2011). The amplification is however smaller in the magnetosheath where the flow decelerates after the bow shock, and the MHD electric-to-magnetic ratio is order 1 in spacecraft frame, as we will see. For the reader possibly surprised by the fact that depending on the sign of $V_{0}$ and of the phase speed $V_{\mathrm{A}}$ of the fluctuations, the ideal-MHD electric field would be zero if $V_{0}=V_{\mathrm{A}}$, consider that this really corresponds to a transformation to a frame moving at $V_{\mathrm{A}}$ in the same direction as the Alfvénic fluctuations, thus at rest with them. It is then expected that the ideal-MHD electric field associated with unidirectional Alfvénic turbulence vanishes in such specific frame. Note that remarkably it is possible to check this result in solar-wind observations where other interesting properties, such as the conservation of magnetic and ion kinetic energy, can be observed in a frame comoving with the - typically antisunward - fluctuations in highly Alfvénic fast speed streams (Matteini et al. 2015).

At small scales and for moderate flow speeds $\left(k d_{\mathrm{i}} \gg 1, V_{0} / V_{\mathrm{A}}\right)$, the electric field of equation (6) is dominated by the non-ideal term $(\propto k)$ and one basically recovers equation (3) also in spacecraft frame. This shows that at sub-ion scales, where magnetic and electric field spectra decouple, the main contribution to the electric field (the non-ideal terms of equation 2) is essentially the same in both spacecraft and plasma frames. In this work then we decide to maintain electric field observations in the spacecraft frame; our choice is motivated by the fact that we are mostly interested in the small-scale behaviour of the spectra. Moreover, while $V_{0} \gg V_{\mathrm{A}}$ in the solar wind, the ratio $V_{0} / V_{\mathrm{A}}$ is not very large in the magnetosheath; therefore, we estimate that this choice does not influence significantly the location of the transition between the fluid and kinetic regimes in spacecraft frame, given by equations (7) and (3), respectively.

\section{DATA ANALYSIS}

We use Cluster data from the Fluxgate Magnetometer (FGM, 22 samples s ${ }^{-1}$; Balogh et al. 1997), the Electric Field and Wave experiment (EFW, 25 samples s $^{-1}$; Gustafsson et al. 1997), and the SpatioTemporal Analysis of Field Fluctuation experiment-Spectrum Analyzer (STAFF-SA, 8 Hz-4 k Hz; Cornilleau-Wehrlin et al. 1997). At full resolution, these instruments ensure a full coverage of spectral frequencies between MHD and electron scales for typical magnetosheath plasma conditions with a switch-over of the detectors at around $10 \mathrm{~Hz}$ (e.g. Alexandrova et al. 2008a). Note that this frequency is roughly one decade larger than the Doppler-shifted typical ion scales at which a spectral break is observed $(0.1-1 \mathrm{~Hz})$. The above instruments measure the three components of the magnetic field and the two components of the electric field in the plane perpendicular to the spacecraft spin axis. In this work, below about $10 \mathrm{~Hz}$, we then calculate wavelet power spectra of the fluctuations of the three-component magnetic field $\delta B$ and of the two-component electric field $\delta E$. However, as discussed by Bale et al. (2005), one of the EFW electric field components ( $x$ in GSE coordinates) is more noisy than the other $(y)$ due to more irregular solar illumination. Since we have verified this condition also in the present data set, in our analysis we have used only $E_{y}$ to characterize electric field spectra at lower frequencies. At higher frequencies, above about $10 \mathrm{~Hz}$, a Fourier analysis of the three-component magnetic field $\delta B$ and of the two-component electric field $\delta E$, as well as a singular value decomposition of the $5 \times 5$ spectral matrix (Santolík, Parrot \& Lefeuvre 2003), gives the intensity and the phase relations of these five components. Following previous studies (Lacombe et al. 2006; Mangeney et al. 2006), the total spectral power of the three-component $\delta B$ and of the two-component $\delta E$, as measured by STAFF-SA, is used, as in this range no significant asymmetry was found between electric field components. We use a selection of intervals presented in Mangeney et al. (2006), corresponding to periods of variable duration, from 1 to $7 \mathrm{~h}$ when Cluster was in the Earth's magnetosheath. Measurements shown are from Cluster 1 (C1) and Cluster 4 (C4). To make a direct comparison between different spectra, magnetic and electric fluctuations have been converted into velocities and all quantities have been normalized to the Alfvén speed $V_{\mathrm{A}}$ as we expect:

$\delta B / B_{0} \sim \delta V / V_{\mathrm{A}} \sim \delta E /\left(V_{\mathrm{A}} B_{0}\right)$

for Alfvénic turbulence. Local values of $V_{\mathrm{A}}$ and $B_{0}$ are indicated for each interval, in order to make it possible to recover spectra in physical units (e.g. $n T^{2} / \mathrm{Hz}$ ) by using relations (8). In the following, frequencies associated with Doppler-shifted particle relevant spatial scales $l$ [taken as $f=V_{0} /(2 \pi l)$ ], namely $f_{\rho \mathrm{p}}$ (proton Larmor radius), $f_{d \mathrm{p}}$ (proton inertial length), $f_{\rho \mathrm{e}}$ (electron Larmor radius), $f_{d \mathrm{e}}$ (electron inertial length), are also shown as reference, as well as the electron cyclotron frequency $f_{\text {ce }}$.

In order to obtain clean spectra at small scales and improve the diagnostic of $\delta E / \delta B$, all STAFF-SA spectra have been inspected to identify and remove the local contribution from local wave activity, like whistlers. These waves are typically observed at electron scales and can be identified by a spectral bump and a signature of circular/elliptical polarization in the plane perpendicular to the magnetic field (Lacombe et al. 2014). We have used the phase difference and the correlation coefficient between the two magnetic field components in the plane perpendicular to the local magnetic field at a given frequency as a diagnostic for whistlers during each of the 4-s spectra measured on board by STAFF-SA. We have separated events (spectra) where a clear whistler signature was detected at any frequencies from those where whistler contribution could be excluded. Only 4-s spectra from the latter case have been averaged and used in the following. An example of the results from the adopted filtering for the magnetic field spectrum is provided in Fig. 1; this interval has the highest level of turbulence among the selected, then allowing for the maximum coverage of small scales. In the top panel, averaged spectra with (blue) and without (red) whistler signatures are shown. The Doppler-shifted frequency $f_{d \mathrm{e}}$ corresponding to the 

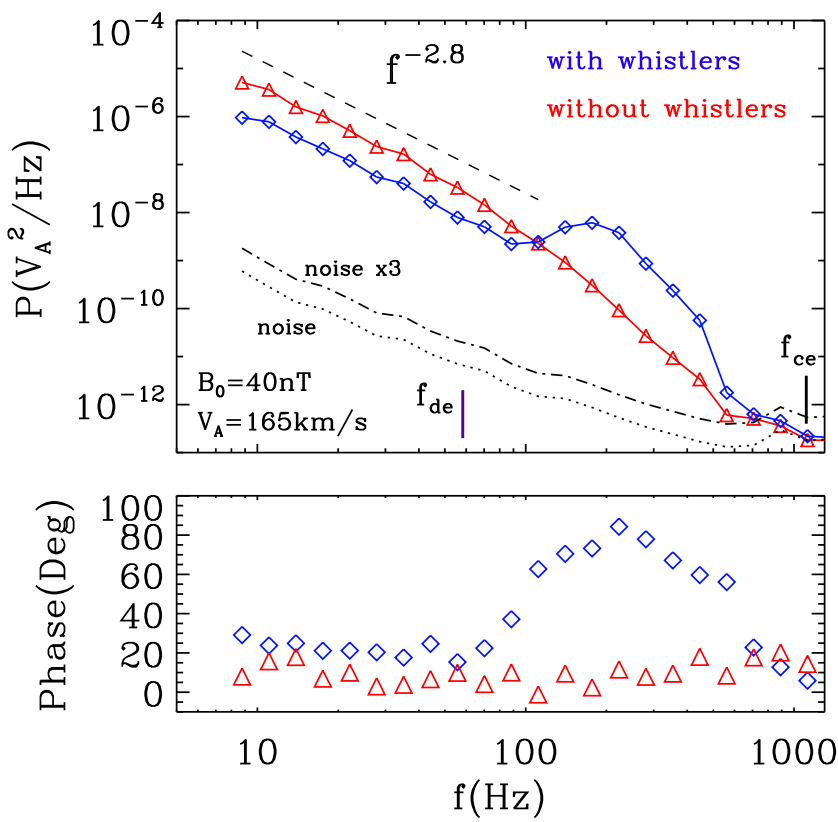

Figure 1. Top: trace power of STAFF-SA mean magnetic spectra selected according to phase difference and coherence between the perpendicular magnetic field components $\left(\delta B_{1,2} \perp B_{0}\right)$ during day 2001-12-16 (03:1509:00 UT) on C4. Curves indicate spectra with (blue) and without (red) whistler wave signatures. The dotted line shows the estimated instrument noise, while the dash-dotted line displays its value multiplied by 3 as a reference. Bottom panel shows the average phase difference angle between magnetic field components in the plane orthogonal to $B_{0}$ for the two sub-sets.

electron inertial length, is shown as a reference, as well as the electron cyclotron frequency $f_{\text {ce. }}$. The estimated noise level of magnetic field measurements of STAFF-SA is also shown as a dotted line, while the dash-dotted line displays its value multiplied by 3 as an estimation of the level at which measurements start to be affected by the noise. At lower frequencies, approximatively below $100 \mathrm{~Hz}$, both spectra show a similar behaviour, consistent with a -2.8 power law typical of the sub-ion regime (e.g. Alexandrova et al. 2009). While above $f_{d e}$, the red curve smoothly steepens until it reaches the level of three times the noise (dash-dotted line) at about $\sim 500 \mathrm{~Hz}$, consistent with turbulent spectra in the solar wind (Sahraoui et al. 2010; Alexandrova et al. 2012), the blue curve (containing whistler waves) has a clear bump around $(0.1-0.5) f_{\text {ce. The }}$ bottom panel displays the phase (in degrees, averaged over spectra) between the two magnetic field components in the plane perpendicular to the mean magnetic field $B_{0}$, as a function of the frequency. This demonstrates that there is a change in the phase difference of the perpendicular magnetic field components at frequencies where the spectral bump is observed. Namely, polarization of the transverse fluctuations passes from linear (phase consistent with $0^{\circ}$ ) to circular $\left(90^{\circ}\right)$. We identify the red curve as the spectrum of the background turbulence; this is the proper spectrum to be used for investigating the turbulent cascade.

Previous analyses (Lacombe et al. 2006; Mangeney et al. 2006) have demonstrated that during these intervals, fluctuations are characterized by quasi-perpendicular $\boldsymbol{k}$-vectors $\left(\boldsymbol{k} \sim \boldsymbol{k}_{\perp}\right)$ between ion and electron scales. Consistent with that and with the divergence free condition, $\boldsymbol{k}_{\perp} \cdot \delta B=0$, no phase difference is found between the two components of $\delta B$ in the plane orthogonal to $\boldsymbol{B}_{0}$ for the turbulent magnetic fluctuations. Conversely, when whistler waves are observed, as they display a well-defined circular polarization in the plane orthogonal to $\boldsymbol{B}_{0}$, this is an indication of quasi-parallel $\boldsymbol{k}$-vectors. From spectra in Fig. 1, the presence of quasiperpendicular $\boldsymbol{k}$-vectors for the purely turbulent spectrum (red) justifies the use of the Taylor hypothesis, since we expect those fluctuations to have low frequencies (cf. Howes, Klein \& TenBarge 2014). We then expect Cluster power spectra to represent Doppler-shifted $\boldsymbol{k}$-vector spectra from MHD to electron scales; consistent with that, the main spectral features discussed in this work are well ordered by spatial scales. The same does not apply for the blue spectrum containing quasi-parallel high-frequency whistler waves, for which the Taylor hypothesis is not satisfied. We can then consider this spectrum as a mixture of spatial (due to relative motion of the plasma with respect to the satellite) and temporal (intrinsic) variations. Consistent with that, the spectral bump related to whistlers is observed beyond $f_{d \mathrm{e}}$ and closer to (but below) the local electron cyclotron frequency $f_{\text {ce }}$, as indeed expected for typical quasi-parallel whistlers (Lacombe et al. 2014; Stansby et al. 2016).

The above analysis demonstrates that a selection of intervals excluding whistler waves is needed in order to correctly infer the properties of sub-ion scale turbulence. Note that when such a separation is not performed (e.g. Huang et al. 2014), some care must probably be used in the interpretation of the results. Moreover, while the example shown in Fig. 1 presents a well-identified and localized bump, this is not always the case. We have found other periods when signatures of circular polarization distinctive of the presence of whistlers appear at various frequencies, resulting in a broad-band contribution. They can therefore not be identified just by visual inspection. The technique used in this work, based on phase inspection, following the analysis of Lacombe et al. (2014), revealed to be suitable for this aim when using STAFF-SA spectra (see also Santolík et al. 2003).

\section{RESULTS AND DISCUSSION}

We start our analysis by addressing properties of the fluctuations at MHD scale and comparing them to more common solar-wind observations. Fig. 2 displays magnetic $E_{B}$ (red) and velocity $E_{V}$ (black) spectra of 2001-12-19 (see also Alexandrova et al. 2008a). This is an approximatively 6-h period (03:15-09:00 UT) with quite steady plasma condition when Cluster was in the flanks of the magnetosheath, enabling a good characterization of large scales. The average angle between the magnetic field and the flow, and proton plasma beta, are $\theta_{B V}=50^{\circ}$ and $\beta_{\mathrm{p}}=1.2$, respectively. Both spectra show a power-law behaviour between $10^{-4}$ and $10^{-1} \mathrm{~Hz}$, indicative of a turbulent cascade. Note that the two spectra also have a slightly different spectral index. The dashed line indicates a power law with spectral index $-3 / 2$, and the dotted line $-5 / 3$. Velocity is approximatively described by the former, while magnetic field by the latter, as is also observed in the inertial range of solar-wind turbulence (Podesta, Roberts \& Goldstein 2007; Salem et al. 2009; Chen et al. 2013b). The bottom panel shows the same spectra compensated by the two powers, confirming the presence of an inertial range (approximatively flat spectra for more than two decades in the magnetic field, slightly less for the proton velocity) and the different index of the two fields. Moreover, in the upper panel, the magnetic field shows a spectral break approaching the estimated frequencies associated with the proton Larmor radius and inertial length, $f_{\rho \mathrm{p}}$ and $f_{d \mathrm{p}}$, respectively, followed by a steeper slope at sub-proton scales, in agreement with typical solar-wind observations (e.g. Leamon et al. 1998; Bruno, Trenchi \& Telloni 2014; Lion, Alexandrova \& Zaslavsky 2016). These properties demonstrate that the turbulent regime discussed here in the magnetosheath has many similarities 


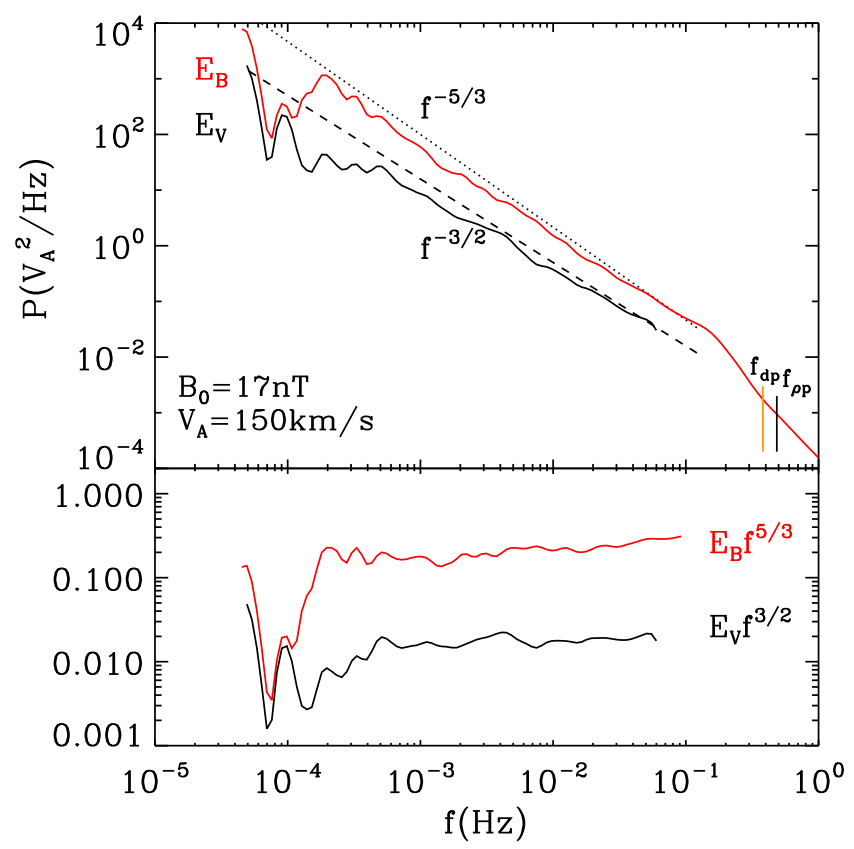

Figure 2. Top panel shows large (MHD) scale magnetic (red) and velocity (black) spectra for day 2001-12-19 (00:00-06:00 UT, C1). Frequencies associated with Doppler-shifted proton scales $f_{d \mathrm{p}}$ (inertial length) and $f_{\rho \mathrm{p}}$ (Larmor radius) are shown as references. Bottom panel shows the same spectra within the inertial range, compensated by $f^{/ / 3}$ and $f^{3 / 2}$, respectively.

with the regime observed in the solar wind within the MHD range. Thanks to the more favourable signal-to-noise ratio, we can now extend the investigation to smaller scales, in a physical range (subion and electron) that cannot currently be fully investigated in the free solar wind.

At kinetic scales, protons are expected to decouple from the magnetic field. At this stage, turbulence is supported by electron-velocity fluctuations rather than the ion ones. These can be retained through the current $\boldsymbol{J} \propto \boldsymbol{v}_{\mathrm{e}}-\boldsymbol{v}_{\mathrm{p}}$, which supports the electric field via the Hall term in equation (1). As shown in Fig. 2, it is not possible to follow the proton-velocity spectrum to larger frequencies with Cluster. However, it is possible to characterize further the turbulent cascade using electric and magnetic spectra. Fig. 3 contains the main result of this work, and shows for the first time composite magnetic $E_{B}$ (red) and electric $E_{\mathrm{E}}$ (blue) field spectra from $\sim 10^{-4}$ to $10^{3} \mathrm{~Hz}$, covering from MHD to electron regimes in plasma turbulence (top panel). The interval used in this case is from 2001-02-12, time 00:15-02:30 UT; during this period, the average plasma beta and angle between the flow and the magnetic field are $\beta_{\mathrm{p}}=1.1$ and $\theta_{B V}=50^{\circ}$, respectively. Instrumental artefacts (spin tones) are visible in the electric field spectrum around $1 \mathrm{~Hz}$, (see also Bale et al. 2005); however, they are quite localized, and do not influence the behaviour at higher frequencies. As in Fig. 1, the black dotted line shows the noise level of STAFF-SA magnetic field. Both magnetic and electric fluctuations show a power law at large scales $(f<0.1 \mathrm{~Hz})$, with a spectral index between $-5 / 3$ and $-3 / 2$ (due to the shorter interval, MHD scales are less well defined than in Fig. 2), and a break close to ion scales. Just after the break, the two spectra shortly decrease with a similar slope, but then they start to depart and the electric field becomes dominant (note that the crossover of the spectra, $\sim 0.5 \mathrm{~Hz}$, occurs well before the beginning of the Spectrum Analyzer range, $8 \mathrm{~Hz}$ ).

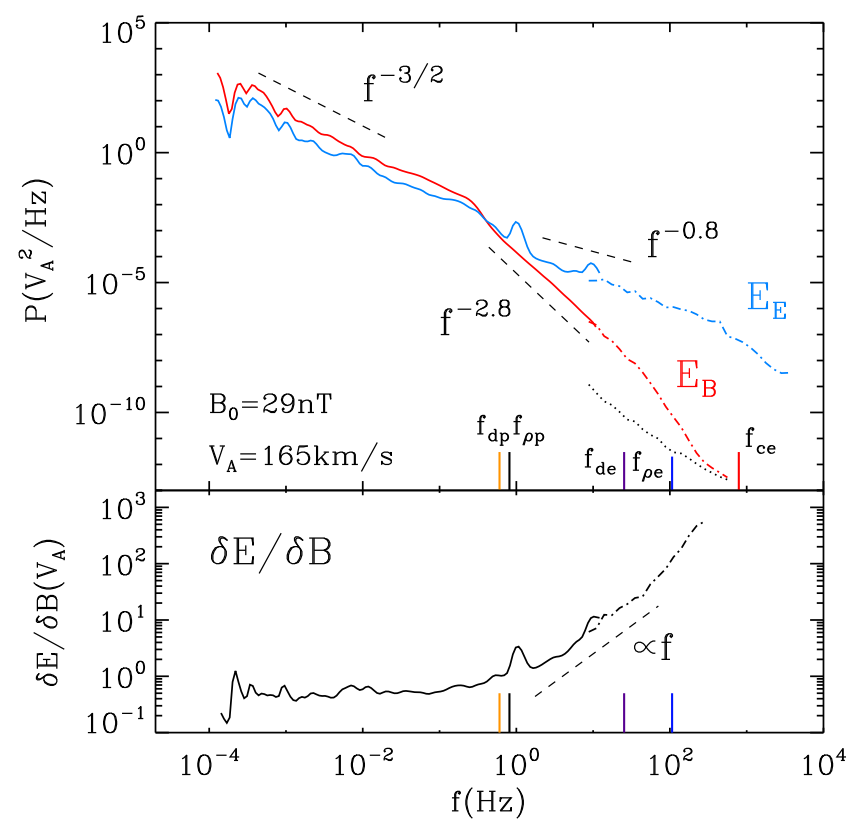

Figure 3. Top: composite FGM/EFW (solid) and STAFF-SA (dash-dotted) spectra of magnetic (red) and electric (blue) field for day 2001-02-12 (00:1502:30 UT, C4). Frequencies associated with ion and electron spatial scales are shown for reference, as well as relevant spectral slopes (dashed lines, see text). Bottom: ratio of electric-to-magnetic amplitude of fluctuations in units of the local Alfvén speed. The linear scaling $\propto f$ predicted by equation (3) between ion and electron scales is shown in dashed line for comparison.

At sub-ion scales, the magnetic field displays the characteristic behaviour observed in solar-wind spectra, consisting in a power law with spectral index close to -2.8 for more than one decade, followed by a steepening when reaching electron scales at $f_{d \mathrm{e}}$ (Alexandrova et al. 2009; Sahraoui et al. 2010). The spectrum reaches the noise level at around $200 \mathrm{~Hz}$. By contrast, the electric field has a shallower spectral slope than the magnetic field at all frequencies beyond ion scales, as expected for the break-down of ideal-MHD. According to equation (2), we expect that in this regime normalized spectra scale as

$\delta E^{2} \propto k^{2} \delta B^{2}$,

implying a relation between electric and magnetic spectral slopes $\alpha$ : $\alpha_{\mathrm{E}}=\alpha_{B}-2$. In Fig. 3, the corresponding spectral slope $\alpha_{\mathrm{E}}=-0.8$ is shown for comparison, leading to a good agreement with the observed sub-proton electric field spectrum. The bottom panel of Fig. 3 shows the electric-to-magnetic ratio of the amplitude of the fluctuations $\delta E / \delta B$ in units of $V_{\mathrm{A}}$ and as a function of the measured frequency. As expected from equation (8) this is order $\sim 1$ and approximatively constant over the whole MHD range, while it increases almost linearly after the ion break, consistently with equation (3). The linear increase $\delta E / \delta B \propto f$ (dashed line) observed between proton and electron scales demonstrates that in this regime the electric field is well described by the generalized Ohm's law and dominated by its non-ideal terms.

To confirm this scenario, more examples are given in Fig. 4, where spectra from four different intervals are shown using the same colour code as in Fig. 3. These correspond to shorter periods with different local plasma beta and angle between magnetic field and plasma flow. Note that the power of the spectrum varies as a function of the angle (Lacombe et al. 2006; Mangeney et al. 2006); moreover 

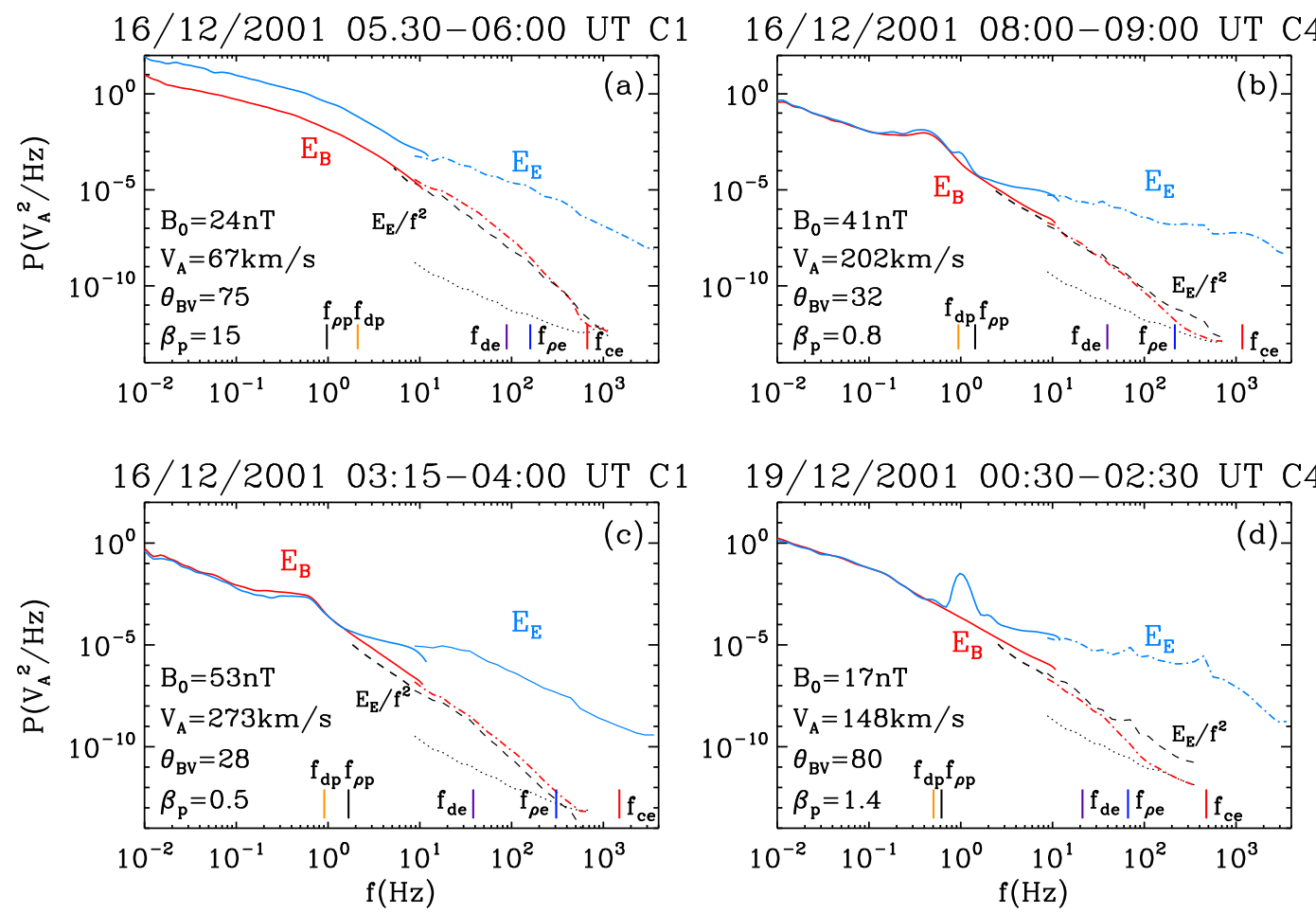

Figure 4. Magnetic and electric spectra for various intervals measured by Cluster in the magnetosheath. Solid (FGM/EFW) and dash-dotted (STAFF-SA) lines show the magnetic (red) and electric (blue) field spectra, as in Fig. 3. Dashed black line shows the expected sub-ion magnetic field contribution estimated from the associated measured electric field multiplied by a factor $f^{-2}$, following to equation (9). The local plasma beta and the angle between the mean magnetic field and the plasma flow are indicated in each panel, as well as frequencies associated with relevant particle scales.

some wave-activity around the ion break could locally influence the spectral slopes, as kinetic instabilities driven by temperature anisotropy are possibly at work as a function of the plasma beta (Samsonov et al. 2007). In the high beta case in the top-left panel, the power of the electric field is higher than magnetic due to a larger $V_{0} / V_{\mathrm{A}}$ ratio (see equation 6). In the bottom-right panel, the matching between the instruments is less good, as FGM measurements are closer to the noise level due to a lower power of the turbulence during this interval. The different scalings of $E$ and $B$ are however well captured by STAFF-SA, as in general for all other intervals. In all panels, the dashed line shows the sub-ion electric field spectrum rescaled as $\tilde{E}_{\mathrm{E}} \propto E_{\mathrm{E}} f^{-2}$ following equation (9); there is a reasonably good agreement between $\tilde{E}_{\mathrm{E}}$ and the observed magnetic field spectrum (red dash-dotted line) down to approximatively $f_{d e}$, confirming that the electric and magnetic field spectra are described by equation (9) in this range. As a consequence, all spectra also display an almost linear evolution of the $\delta E / \delta B$ ratio (not shown), consistent with the bottom panel of Fig. 3 .

A final remark should be made on the behaviour of the spectra at sub-electron scales $\left(k \rho_{\mathrm{e}}>1\right)$. The magnetic field spectrum reaches the noise after $f_{\rho \mathrm{e}}$ and is not suitable for investigation at smaller scales. On the other hand, the electric field spectrum is higher than the antenna noise at all frequencies (see Mangeney et al. 2006, for details), although it does not provide a clear insight of the sub-electron trend due to the large variability among different intervals. As a general feature, the electric field spectral slope tends to become steeper around the electron cyclotron frequency $f_{\text {ce }}(f \gtrsim 500 \mathrm{~Hz})$ : see Figs 3 and 4 . In this range, also electrons decouple from the magnetic field and the use of the generalized Ohm's law of equation (1) is no more suitable; fluctuations are expected to become purely electrostatic. Previous investigations of these data periods (Lacombe et al. 2006; Mangeney et al. 2006) have highlighted that, while at larger scales $\left(k \rho_{\mathrm{e}} \lesssim 10\right)$ the electric field power is dominated by quasi-perpendicular $\boldsymbol{k}$-vectors $E\left(\boldsymbol{k}_{\perp}\right)>$ $E\left(\boldsymbol{k}_{\|}\right)$of electromagnetic fluctuations, at higher frequencies it seems to be dominated by quasi-parallel $\boldsymbol{k}$-vectors $E\left(\boldsymbol{k}_{\|}\right)>E\left(\boldsymbol{k}_{\perp}\right)$ of electrostatic activity (see figs 4 and 8 of Mangeney et al. 2006).

\section{CONCLUSIONS}

In summary, we have shown for the first time magnetic and electric field spectra from MHD to electron scales in a space plasma. While such a measurement is not achievable in the free solar wind using current spacecraft, due to the high noise level with respect to the typical power of electric field fluctuations at sub-ion scales (cf. Bale et al. 2005; Salem et al. 2012), this investigation is possible in the magnetosheath, downstream of the Earth's bow shock, using Cluster data (Mangeney et al. 2006; Sundkvist et al. 2007). Our results confirm that in the transition from MHD to kinetic plasma turbulence, the slope of electric- and magnetic field spectra depart around ion scales, with the former following a shallower slope than the latter. Such a behaviour is consistent with electric field fluctuations supported by the non-ideal terms in the Ohm's law, and is recovered also in simulations performed with different numerical models but sharing basic aspects of equation (1) (e.g. Howes et al. 2011; Passot et al. 2014; Valentini et al. 2014; Franci et al. 2015a). Moreover, the observed scaling of the electric-to-magnetic ratio $\delta E / \delta B \propto f$ between proton and electron scales is in very good agreement with the theoretical expectation (equation 6).

These results, so far restricted to the magnetosheath, are expected to reflect a more universal scaling in kinetic plasma turbulence which could be tested with future spacecraft in other environments. Note that favourable conditions for the study of small-scale electric and magnetic fields can also be found in local and transient events 
in space plasmas, as for example suggested by Cluster measurements of turbulent spectra within reconnection exhausts (Eastwood et al. 2009), THEMIS observations of bursty bulk flows in Earth's plasma sheet (e.g. Chaston et al. 2012; Ergun et al. 2015) and, more recently, MMS observations of turbulence within KelvinHelmholtz vortices at Earth's magnetopause (Stawarz et al. 2016). Moreover, these findings confirm the need for accurate and highresolution simultaneous measurements of magnetic and electric fields in order to capture the processes responsible for turbulent energy dissipation and particle heating in space plasmas, as aimed in the THOR mission proposal ${ }^{1}$ (Vaivads et al. 2016).

\section{ACKNOWLEDGEMENTS}

The authors acknowledge very stimulating and helpful discussions with A. Schekochihin, S. Landi, A. Verdini, L. Franci, and P. Hellinger. This work was supported by Science and Technology Facilities Council (STFC) grant ST/N000692/1. CHKC was supported by an STFC Ernest Rutherford Fellowship and an Imperial College Junior Research Fellowship.

The authors thank the PIs of STAFF (N. Cornilleau-Wehrlin), FGM (A. Balogh and E. Lucek), EFW (G. Gustafsson), CIS (H. Rème, I. Dandouras) instruments and the Cluster Science Archive (ESA). The French contribution to the Cluster project has been supported by the European Space Agency (ESA) and by the Centre National d'Etudes Spatiales (CNES).

\section{REFERENCES}

Alexandrova O., Saur J., 2008, Geophys. Res. Lett., 35, L15102

Alexandrova O., Mangeney A., Maksimovic M., Cornilleau-Wehrlin N., Bosqued J., André M., 2006, J. Geophys. Res., 111, 12208

Alexandrova O., Lacombe C., Mangeney A., 2008a, Ann. Geophys., 26, 3585

Alexandrova O., Carbone V., Veltri P., Sorriso-Valvo L., 2008b, ApJ, 674, 1153

Alexandrova O., Saur J., Lacombe C., Mangeney A., Mitchell J., Schwartz S. J., Robert P., 2009, Phys. Rev. Lett., 103, 165003

Alexandrova O., Lacombe C., Mangeney A., Grappin R., Maksimovic M., 2012, ApJ, 760, 121

Alexandrova O., Chen C. H. K., Sorriso-Valvo L., Horbury T. S., Bale S. D., 2013, Space Sci. Rev., 178, 101

Bale S. D., Kellogg P. J., Mozer F. S., Horbury T. S., Reme H., 2005, Phys. Rev. Lett., 94, 215002

Balogh A. et al., 1997, Space Sci. Rev., 79, 65

Boldyrev S., Horaites K., Xia Q., Perez J. C., 2013, ApJ, 777, 41

Bruno R., Carbone V., 2013, Living Rev. Sol. Phys., 10, 2

Bruno R., Trenchi L., Telloni D., 2014, ApJ, 793, L15

Cerri S. S., Califano F., Jenko F., Told D., Rincon F., 2016, ApJ, 822, L12

Chaston C. C., Bonnell J. W., Clausen L., Angelopoulos V., 2012, J. Geophys. Res., 117, A12205

Chen C. H. K., Horbury T. S., Schekochihin A. A., Wicks R. T., Alexandrova O., Mitchell J., 2010, Phys. Rev. Lett., 104, 255002

Chen C. H. K., Bale S. D., Salem C., Mozer F. S., 2011, ApJ, 737, L41

Chen C. H. K., Salem C. S., Bonnell J. W., Mozer F. S., Bale S. D., 2012, Phys. Rev. Lett., 109, 035001

Chen C. H. K., Boldyrev S., Xia Q., Perez J. C., 2013a, Phys. Rev. Lett., 110,225002

Chen C. H. K., Bale S. D., Salem C. S., Maruca B. A., 2013b, ApJ, 770, 125

Chen C. H. K., Leung L., Boldyrev S., Maruca B. A., Bale S. D., 2014, Geophys. Res. Lett., 41, 8081

Cho J., Lazarian A., 2004, ApJ, 615, L41

Cornilleau-Wehrlin N. et al., 1997, Space Sci. Rev., 79, 107
Eastwood J. P., Phan T. D., Bale S. D., Tjulin A., 2009, Phys. Rev. Lett., 102,035001

Ergun R. E., Goodrich K. A., Stawarz J. E., Andersson L., Angelopoulos V., 2015, J. Geophys. Res., 120, 1832

Franci L., Verdini A., Matteini L., Landi S., Hellinger P., 2015a, ApJ, 804, L39

Franci L., Landi S., Matteini L., Verdini A., Hellinger P., 2015b, ApJ, 812, 21

Franci L., Landi S., Matteini L., Verdini A., Hellinger P., 2016, ApJ, 833, 91

Gustafsson G. et al., 1997, Space Sci. Rev., 79, 137

Hadid L. Z., Sahraoui F., Kiyani K. H., Retinò A., Modolo R., Canu P., Masters A., Dougherty M. K., 2015, ApJ, 813, L29

Howes G. G., Tenbarge J. M., Dorland W., Quataert E., Schekochihin A. A., Numata R., Tatsuno T., 2011, Phys. Rev. Lett., 107, 035004

Howes G. G., Klein K. G., TenBarge J. M., 2014, ApJ, 789, 106

Huang S. Y., Sahraoui F., Deng X. H., He J. S., Yuan Z. G., Zhou M., Pang Y., Fu H. S., 2014, ApJ, 789, L28

Kiyani K. H., Chapman S. C., Sahraoui F., Hnat B., Fauvarque O., Khotyaintsev Y. V., 2013, ApJ, 763, 10

Kunz M. W., Schekochihin A. A., Stone J. M., 2014, Phys. Rev. Lett., 112, 205003

Lacombe C., Samsonov A. A., Mangeney A., Maksimovic M., CornilleauWehrlin N., Harvey C. C., Bosqued J.-M., Trávníček P., 2006, Ann. Geophys., 24, 3523

Lacombe C., Alexandrova O., Matteini L., Santolík O., Cornilleau-Wehrlin N., Mangeney A., de Conchy Y., Maksimovic M., 2014, ApJ, 796, 5

Leamon R. J., Smith C. W., Ness N. F., Matthaeus W. H., Wong H. K., 1998, J. Geophys. Res., 103, 4775

Lion S., Alexandrova O., Zaslavsky A., 2016, ApJ, 824, 47

Mangeney A., Lacombe C., Maksimovic M., Samsonov A. A., CornilleauWehrlin N., Harvey C. C., Bosqued J.-M., Trávníček P., 2006, Ann. Geophys., 24, 3507

Matteini L., Horbury T. S., Pantellini F., Velli M., Schwartz S. J., 2015, ApJ, 802,11

Passot T., Henri P., Laveder D., Sulem P.-L., 2014, Eur. Phys. J. D, 68, 207

Podesta J. J., Roberts D. A., Goldstein M. L., 2007, ApJ, 664, 543

Rappazzo A. F., Velli M., Einaudi G., 2013, ApJ, 771, 76

Šafránková J., Němeček Z., Přech L., Zastenker G. N., 2013, Phys. Rev. Lett., 110, 025004

Sahraoui F., Belmont G., Rezeau L., Cornilleau-Wehrlin N., Pinçon J. L., Balogh A., 2006, Phys. Rev. Lett., 96, 075002

Sahraoui F., Goldstein M. L., Robert P., Khotyaintsev Y. V., 2009, Phys. Rev. Lett., 102, 231102

Sahraoui F., Goldstein M. L., Belmont G., Canu P., Rezeau L., 2010, Phys. Rev. Lett., 105, 131101

Salem C., Mangeney A., Bale S. D., Veltri P., 2009, ApJ, 702, 537

Salem C. S., Howes G. G., Sundkvist D., Bale S. D., Chaston C. C., Chen C. H. K., Mozer F. S., 2012, ApJ, 745, L9

Samsonov A. A., Alexandrova O., Lacombe C., Maksimovic M., Gary S. P., 2007, Ann. Geophys., 25, 1157

Santolík O., Parrot M., Lefeuvre F., 2003, Radio Sci., 38, 10

Schekochihin A. A., Cowley S. C., Kulsrud R. M., Hammett G. W., Sharma P., 2005, ApJ, 629, 139

Schekochihin A. A., Cowley S. C., Dorland W., Hammett G. W., Howes G. G., Quataert E., Tatsuno T., 2009, ApJS, 182, 310

Stansby D., Horbury T. S., Chen C. H. K., Matteini L., 2016, ApJ, 829, L16

Stawarz J. E. et al., 2016, J. Geophys. Res. Space Phys., 121, 11021

Sundkvist D., Retinò A., Vaivads A., Bale S. D., 2007, Phys. Rev. Lett., 99, 025004

Vaivads A. et al., 2016, Journal of Plasma Physics, 82, 905820501

Valentini F., Servidio S., Perrone D., Califano F., Matthaeus W. H., Veltri P., 2014, Phys. Plasmas, 21, 082307

von Papen M., Saur J., Alexandrova O., 2014, J. Geophys. Res., 119, 2797

Zimbardo G., Greco A., Sorriso-Valvo L., Perri S., Vörös Z., Aburjania G., Chargazia K., Alexandrova O., 2010, Space Sci. Rev., 156, 89 